\title{
First setup for cooled GaAs cathodes with increased charge lifetime*
}

\author{
T. Eggert ${ }^{\dagger}$, J. Enders, M. Espig, Y. Fritzsche, N. Kurichiyanil, M. Wagner, S. Weih \\ Institut für Kernhysik, TU Darmstadt, Germany \\ E-mail: teggerteikp.tu-darmstadt.de,
}

GaAs photocathodes can be used for generation of highly polarized electron beams. For highcurrent applications it is necessary to maximize the charge lifetime of the cathode material to ensure reliable operation. It is expected to increase the local vacuum conditions by cryogenic cooling of the electrode and a local subvolume, due to cryogenic adsorption of reactive residual gas molecules at the surrounding walls. This both protects the sensitive negative-electron-affinity surface of the cathode and allows a higher laser power deposited in the material, resulting in higher possible beam currents. An electrostatic bend is introduced to reduce Ion-backbombardment and further increase the cathode lifetime. To measure the characteristics of such a cryogenic source, a dedicated set-up is being developed at the Photo-CATCH test facility in Darmstadt, Germany.

XVII International Workshop on Polarized Sources, Targets and Polarimetry

Kaist, South Korea

16-20 October, 2017

${ }^{*}$ Work supported by DFG (GRK 2128) and BMBF (05H15RDRB1). This proceeding is an update to [1].

${ }^{\dagger}$ Speaker. 


\section{Introduction}

For the generation of high-intensity polarized electron beams for ERL experiments [2], positron production [3], or future colliders in general [4], photo-guns can be used as high-current, low emittance electron sources. GaAs cathodes, however exhibit limited lifetime due to the sensitivity of the negative-electron affinity (NEA) layer necessary for electron emission. In this work two lifetime limiting effects are being addressed. The vacuum lifetime is mainly limited by chemical reactions of oxygen containing residual gases with the NEA layer. While in operation the accelerated electrons ionize residual gas molecules, which are accelerated back towards the cathodes. This ion-backbombardment (IBB) called effect has to be reduced to increase the charge lifetime [5].

To increase the local vacuum conditions the cathode is installed in a cryogenic environment, which provides a cryopumping effect at the cold surfaces to reduce the pressure. In addition the cathode itself will be cooled, which reduces the heat load induced by the laser. To further reduce the IBB an electrostatic bend of the electron beam is introduced. By means of a cryogenic source design described in this article, it could be therefore possible to achieve greater cathode lifetimes compared to conventional semiconductor-based photo-electron guns.

\section{Increasing Cathode Lifetime}

The lifetime of GaAs photocathodes is limited by the lifetime of the negative electron affinity (NEA) layer applied to the GaAs surface to achieve high quantum efficiency (QE). It can be represented as [6]

$$
\frac{1}{\tau}=\sum_{i} \frac{1}{\tau_{i}}=\frac{1}{\tau_{v a c}}+\frac{1}{\tau_{f e}}+\frac{1}{\tau_{l o s s}}+\frac{1}{\tau_{i b b}}
$$

where $\tau$ is the total lifetime of the cathode, $\tau_{v a c}$ is the vacuum lifetime, $\tau_{f e}$ the lifetime due to field emission, $\tau_{\text {loss }}$ describes the lifetime related to beam loss, and $\tau_{i b b}$ describes the destruction of the NEA layer due to ion back bombardment .

Field emission and beam loss effects are determined by the geometry and voltage layout of the source. The vacuum lifetime and ion back bombardment are directly related to the vacuum conditions.

The lifetime in vacuum is mainly limited by chemical reactions of the $\mathrm{CsO}$ layer with residual gas molecules, which corrode the layer over time. It has been shown [7] that molecules containing oxygen strongly affect the vacuum lifetime. However, at temperatures around and below $10 \mathrm{~K}$, which will be achieved with the setup described below, gases such as $\mathrm{O}_{2}, \mathrm{CO}_{2}$, or $\mathrm{CO}$ do not play a role anymore, and the residual gas pressure is dominated by $\mathrm{H}_{2}$ and $\mathrm{He}$ [8]. Thus cryocooling would directly contribute to an increased vacuum lifetime. Preliminary estimates of the vacuum conditions inside an almost completely closed cryogenic subvolume show that pressures of a factor $10^{-3}$ lower compared to the outer chamber can be achieved.

The outgassing of $\mathrm{H}_{2}$ cannot be prevented completely at the expected temperature. However this has no effect on the vacuum lifetime, since $\mathrm{H}_{2}$ does not corrode the cathode [7]. But the 

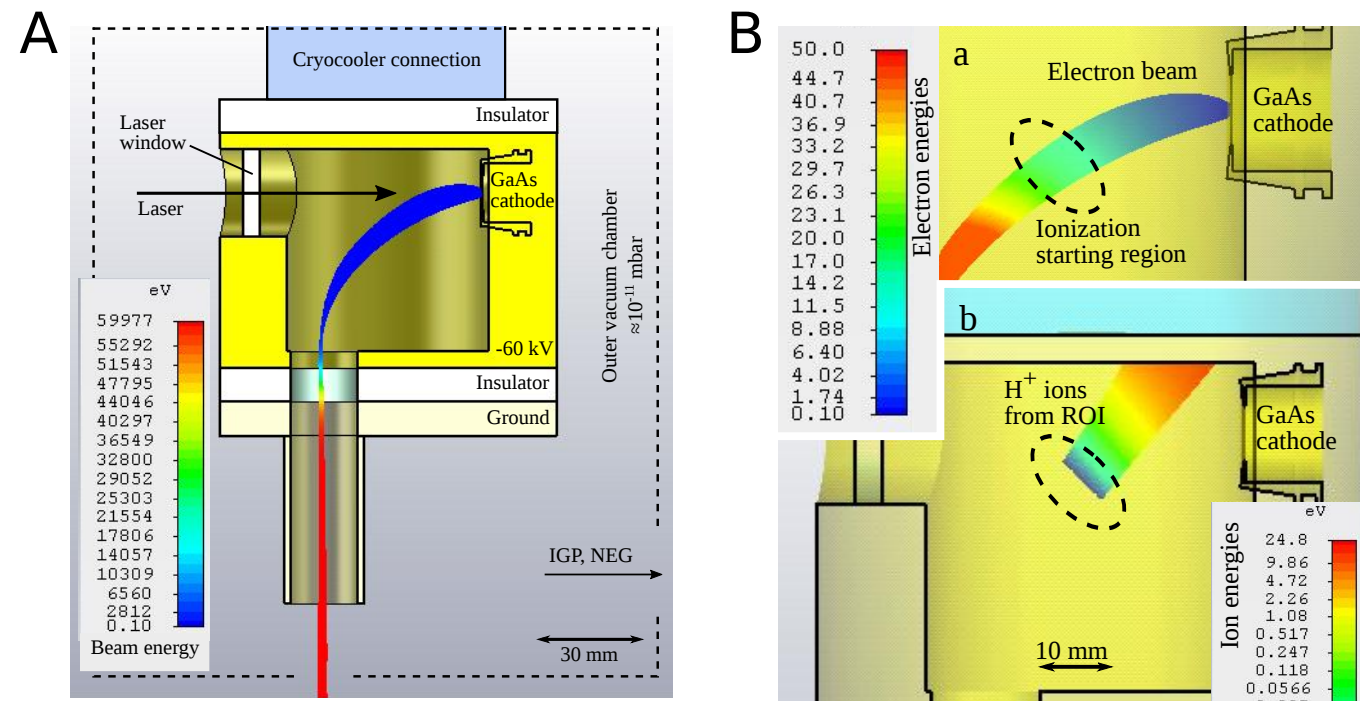

Figure 1: A: Simulation of the electron beam with a first layout of the cryogenic source (CST studio suite $($ )). $\mathrm{B}$ : The region within the electrode chamber where the electron beam reaches sufficient energies to ionize molecular hydrogen (a). Ions originating from this ROI travel back a different path than the electrons and miss the GaAs cathode (b), presuming they have no initial kinetic energy.

presence of $\mathrm{H}_{2}$ contributs to the IBB. To reduce the destruction of the CsO layer due to IBB, an electrostatic bend, which deflects the electrons away from the incident laser axis is introduced. Due to the higher mass, the ionized gas molecules take a different path, and therefore miss the cathode.

\section{Source Design}

Fig. 1A shows a preliminary layout of the cryo source. An almost completely closed cryogenic subvolume houses the GaAs cathode. Cooling of the cryo-volume is provided by a cryocooler attached on the top of the chamber. The only conduction to the warm outside volume is provided by the beam tube, which reduces the flow of molecules from the outside volume to the cooled inner surface of the source. This prevents the contamination of the cold surface, which would reduce the cryopumping effect. To protect the cryocooler from the electrode potential it has to be separated from the chamber by an insulator.

The cooled subvolume is placed inside a lager vacuum chamber, which is connected to a combination of different pumps to achieve pressures in the range of $10^{-11} \mathrm{mbar}$ at room temperature. A modified puck ensures proper thermal contact of the GaAs cathode with the electrode chamber for sufficient cathode cooling.

Fig. 1B shows a simulation of the path of ionized gas molecules. Significant electron impact ionization of $\mathrm{H}_{2}$ occurs at electron energies from $15.6 \mathrm{eV}$ to around $1 \mathrm{keV}$ [9]. As it can be seen, ions originating from the region where the electron beam reaches sufficient ionization energies are already missing the cathode due to the electrostatic bend. Therefore, direct IBB is expected to be prevented almost completely with the new design. 
In the current design, a voltage of $-60 \mathrm{kV}$ is applied to the electrode chamber. However, further simulations of different chamber geometries and voltages are planned to study the influence on the beam quality.

The inner chamber will be manufactured from aluminum. This ensures low outgassing rates for optimal vacuum conditions. The outer surface of the chamber will be polished to achieve a low emissivity to minimize radiation heat load and ensures a stable operation at cryogenic temperatures. For the insulator material $\mathrm{Al}_{2} \mathrm{O}_{3}$ is very well suited. It provides a high thermal conductivity at cryogenic temperatures as well as a high dielectric strength.

\section{Conclusion and Outlook}

By placing the GaAs photocathode in a cryogenic, confined subvolume and using an electrostatic bend for IBB reduction, the overall cathode lifetime is expected to be increased significantly compared to conventional sources. This would enable high-current applications of spin-polarized electron beams.

For optimal beam quality, alternative chamber geometries and different potentials will be tested in simulations. After the implementation, first measurements are planned to be conducted at the Darmstadt photo-CATCH test set-up [10] to investigate the lifetime as well as the quantum efficiency of the new source.

\section{References}

[1] S. Weih, T. Eggert, et al., "Development of a Cryogenic GaAs DC Photo-Gun for High-Current Applications", in Proc. 8th Int. Particle Accelerator Conf. (IPAC'17), Copenhagen, Denmark, May 2017, paper TUPAB032, pp. 1391-1392, https://doi.org/10.18429/JACoW-IPAC2017-TUPAB032, 2017.

[2] C. K. Sinclair, "DC photoemission electron guns as ERL sources", Nucl. Inst. Meth. A, vol. 557, pp. 69-74, 2006.

[3] D. Abbot et al. (PEPPO Collaboration), "Production of Highly Polarized Positrons Using Polarized Electrons at MeV Energies", Phys. Rev. Lett., vol. 116, 214801, 2016.

[4] A. Brachmann et al., "The Polarized Electron Source for the International Collider (ILC) Project", in AIP conf. Proc., vol. 915, pp. 1091-1094, 2006.

[5] C. K. Sinclair et al. , "Development of a high average current polarized electron source with long cathode operational lifetime", Phys. Rev. ST Accel. Beams 10, 023501, 2007

[6] K. Aulenbacher, G. Arz, R. Barday and V. Tioukine, "Photocathode Life Time Research at MAMI", in Proc. SPIN 2004, pp. 975-979, 2005.

[7] N. Chanlek et al., "The degradation of quantum efficiency in negative electron affinity GaA photocathodes under gas exposure”, J. Phys D: Appl. Phys., vol. 47, 055110, 2014.

[8] C. Day, Cern Report 10.5170/CERN-2007-003.241, Basics and applications of cryopumps, 2007.

[9] L. J. Kieffer and G. H. Dunn, "Electron Impact Ionization Cross-Section Data for Atoms, Atomic Ions, and Diatomic Molecules: I. Experimental Data", Rev. Mod. Phys., vol. 38, 1, 1966.

[10] N. Kurichiyanil, "Design and construction of a test stand for photocathode research an experiments", Dissertation, Technische Universität Darmstadt, Darmstadt, Germany, 2017. 